\title{
Bioelectric Potentials of Plant for Determining Human Positions
}

\author{
Imam Tahyudin ${ }^{1,2}$ and Hidetaka Nambo ${ }^{1^{*}}$ \\ ${ }^{1}$ Artificial Intelligence Laboratory, Graduate School of Natural Science and Technology \\ Division of Electrical Engineering and Computer Science, Kanazawa University, \\ Kakuma, Kanazawa, Ishikawa 920-1192, Japan \\ ${ }^{2}$ Department of Information Systems, STMIK AMIKOM Purwokerto, \\ J1. Letjendpol Soemarto, Purwokerto, Central Java 35127, Indonesia \\ (Received December 31, 2017; accepted February 20, 2018)
}

Keywords: natural sensor, bioelectric potential plant, position estimation, MOPAR

The use of plants as biological monitors to detect human behavior is very interesting to investigate. One benefit is to know the position of elderly people living alone in a house in order to avoid accidents resulting in death because help is not immediate. In this study, we use an association analysis with the particle swarm optimization approach. This method is called MOPAR or multi-objective of particle swarm optimization for numerical association rule mining. Real data sets of the bioelectric potentials of plants were used to obtain rules and to examine the accuracy of the method. This method shows promising results.

\section{Introduction}

That the bioelectric potential of plants may be used as a natural sensor is an innovative effort to monitor human behavior. ${ }^{(1-5)}$ For example, consider accidents involving elderly people who live alone in a home: they do not often receive help immediately. Previous attempts to monitor activities using cameras, however, were rejected because the monitor might be placed in a private room such as a bathroom or a bedroom. ${ }^{(2-5)}$ In addition, infrared sensors were tested to address this problem and, even though the results were good, the cost was high because capturing human behavior required many sensors, so the method was not economically feasible. ${ }^{(6)}$ Other solutions used the sense of smell, but the results were not good because there was too much noise in the data. ${ }^{(6)}$ Hence, the use of the bioelectric potential of plants could be a solution to these problems because plants are a welcome addition to private places, low in cost, and they are beneficial to health because they produce oxygen, which reduces stress and provides a sense of freshness. ${ }^{(2-5)}$

From the results of previous studies, the bioelectric potential of plants has the ability to capture human behavior well. Research conducted by Shimbo et al. has shown that human behavior such as touching a plant, opening a door, approaching a plant, and turning on the light can be detected by extracting the characteristic of bioelectrical potential of plants. ${ }^{(7)}$

*Corresponding author: e-mail: nambo@blitz.ec.t.kanazawa-u.ac.jp

http://dx.doi.org/10.18494/SAM.2018.1887 
Subsequent research conducted by Jin et al. used an artificial neural network algorithm to successfully detect the distance of a person from a plant by observing the plant's bioelectric potential. $^{(6)}$ Another study conducted by Nambo et al. used the bioelectric potential of plants to estimate whether people were in a room. They used several algorithms including a decision tree (J48) for classification and a multilayer perceptron to determine the presence of people. Next, they carried out a regression model for a matching process. The results showed that a person's presence in a room could be determined with an accuracy of $60 \% .{ }^{(3-5)}$ These previous research studies did not determine the specific position of people in a given space. Therefore, in this research, we aimed to estimate the exact position of people using the bioelectric potential of plants by the association rule mining with particle swarm optimization (PSO).

This method is a multi-objective particle swarm optimization algorithm for solving a numerical association rule mining problem (MOPAR). The proposed method was widely used in various fields such as for the completion of work job scheduling problems, to evaluate the stock market, to infer gene regulatory networks, and for the optimization of a numerical association analysis problem. ${ }^{(8-11)}$ The MOPAR method has more advantages than decision trees or the deep learning method. One of them is that we can see the rules generated by the MOPAR method. On the other hand, deep learning is a black box method, so we cannot see any rules as a result.

The research is organized as follows. In Sect. 2, we present the proposed method for determining object position and explain the experimental design. In Sect. 3, we present the bioelectric potential data set, its analysis, the matching process, and its accuracy. Finally, Sect. 4 is the conclusion.

\section{Proposed Methods}

\subsection{Measurement of bioelectric potential of plants}

Measurements are carried out using the data logger GRAPHTEC GL400-4. It measures low voltage at a sampling rate of $512 \mathrm{~Hz}$. We use this sampling rate because we expect the potential change to be within $1 \mathrm{~s}$ at most, which is regarded as 1 unit analysis of data. This tool has four channels so it can simultaneously measure voltage.

For the measurement of the bioelectric potential of plants, electrodes are attached on two different leaves and the voltage generated between both leaves is measured. The measurements are stored in a PC. In this experiment, no ground is used as the electrical potential. In Jin's study, he used the soil of the plant as the ground. ${ }^{(6)}$ However, it seems that the soil is also influenced by something and it is not good as the ground. We think it is influenced by the root of the plant. We considered the ground, but in this study, we observed the difference in electrical potential between the leaves.

Figure 1 shows the plant connected to the data logger and the PC. The signal recording is shown in Fig. 2. 


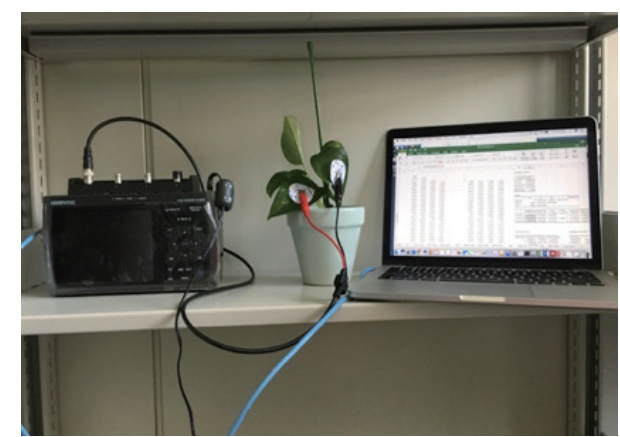

Fig. 1. (Color online) Measuring the bioelectric potential of a plant.

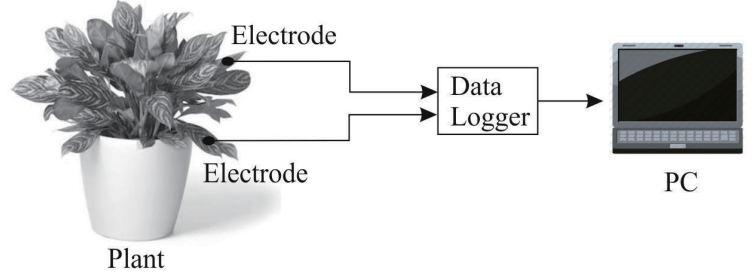

Fig. 2. Illustration of signal recording.

\subsection{MOPAR}

MOPAR is a method introduced by Beiranvand et al. in 2014, who also developed the PSO method with numerical association analysis. ${ }^{(11)}$ PSO was first introduced by Kennedy and Eberhart in 1995. ${ }^{(12)}$ It is an evolutionary method inspired by animal behavior such as the flocking of birds, the schooling of fish, and the swarming of bees. ${ }^{(12)}$

The main model of PSO is given in Eqs. (1) and (2). The first equation is the velocity model and the second one is the position model. ${ }^{(12)}$

$$
\begin{gathered}
v_{i}^{\text {new }}=\omega v_{i}^{\text {old }}+c_{1} \text { rand }()\left(\text { pBest }-x_{i}\right)+c_{2} \operatorname{rand}()\left(\text { Best }-x_{i}\right) \\
x_{i}^{\text {new }}=x_{i}^{\text {old }}+v_{i}^{\text {new }}
\end{gathered}
$$

Here, $\omega$ is the inertial weight, $i$ represents the number of particles, $v_{i}^{\text {old }}$ and $v_{i}^{\text {new }}$ are the particle velocities of the particle $i$ before and after the updating process, respectively, $x_{i}$ is the position of the first or most recent position, $\operatorname{rand}()$ is a random variable in the range of $(0,1)$ following a uniform function, $c_{1}$ and $c_{2}$ are a cognitive component and a social component, pBest is the best particles in a local position, and gBest is the best solution globally from the whole position. Velocity in each dimension is limited to a maximum velocity $\left(v_{\max }\right)$ to optimize the time of the search process..$^{(12,13)}$

\subsection{Experimental design}

In this section, we describe the method used to make the rules for the location of three objects by using MOPAR. The rules are used in a matching process to determine the position of people. The experimental design is shown in Fig. 3.

Figure 3 is explained below.

1. Data set preparation

The bioelectric potential data set is obtained from the positions of the three objects and two plants after calculating the cepstrum. 


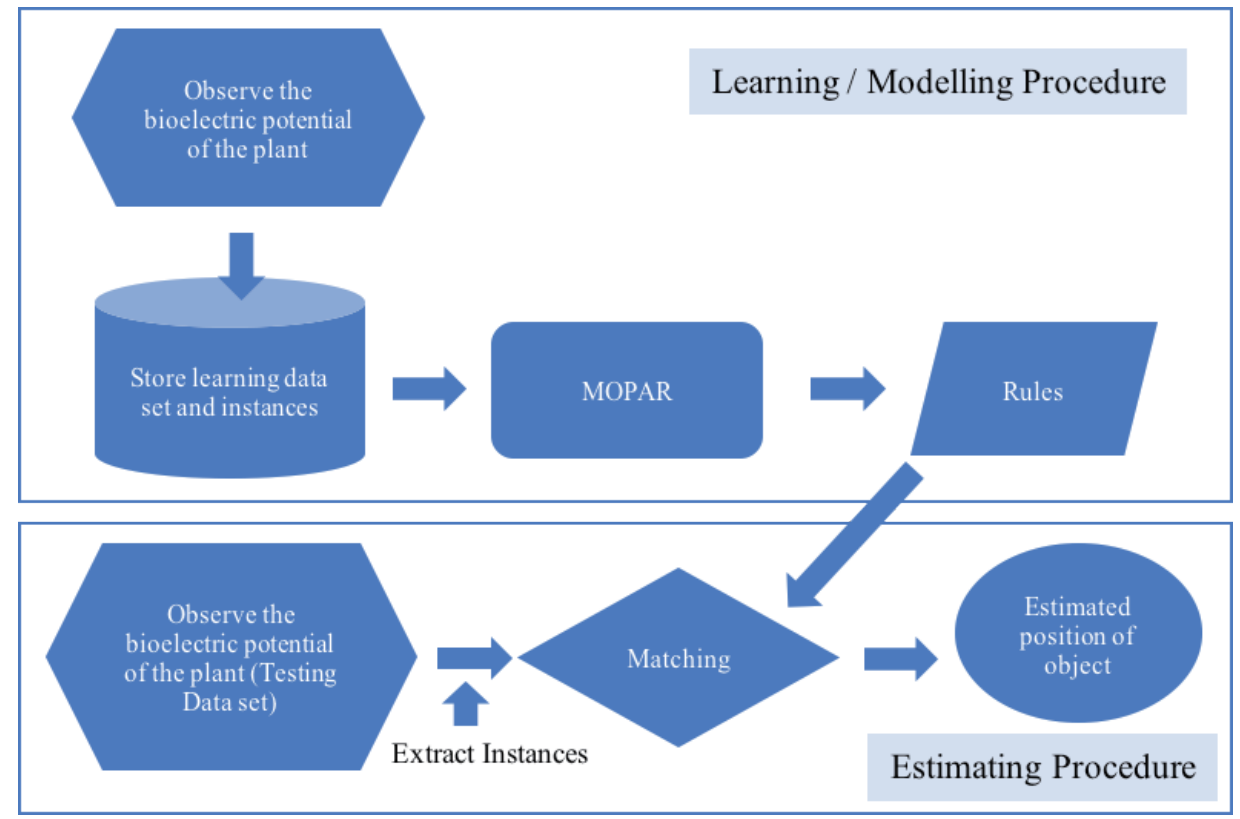

Fig. 3. (Color online) Experimental design.

2. MOPAR analysis

This step runs the MOPAR algorithm for the position of every object. The output of this process is the set of rules used for the matching process.

3. Matching process

This process matches rules with data set testing from the position of every object.

4. Evaluation

To confirm the estimation of object position, we calculate the accuracy. This process applies the matching process for each block. After that, the number of blocks that match in accordance with the rules is shown.

\section{Experiments and Results}

\subsection{Setting up the analysis parameters}

The experiment uses a MacBook Pro with these specifications: $2.7 \mathrm{GHz}$ Intel Core i5, 8 GB $1867 \mathrm{MHz}$, and DDR3. The analysis uses Matlab software. We set up the parameters such as population size, the size of external storage, the number of iterations, the values of $c_{1}$ and $c_{2}, \omega$, xRank, and velocity limits. The parameter values are listed in Table 1.

\subsection{Bioelectric potential data set}

The experiment was conducted in a $5.75 \times 3.45 \mathrm{~m}^{2}$ room with three object positions, M1, M2, and M3, and two plants, P1 and P2. A person walked around each object for $30 \mathrm{~s}$. Two plants detect a response to human action, and the changes in the data were seen on the monitor 
Table 1

Setup parameters.

\begin{tabular}{ccccccc}
\hline $\begin{array}{c}\text { Population } \\
\text { size }\end{array}$ & $\begin{array}{c}\text { External } \\
\text { repository size }\end{array}$ & $\begin{array}{c}\text { Number of } \\
\text { iterations }\end{array}$ & $c_{1}$ and $c_{2}$ & $\omega$ & $\begin{array}{c}\text { Velocity } \\
\text { limit }\end{array}$ & xRank \\
\hline 40 & 100 & 700 & 2 & 0.9 & 3.83 & 13.33 \\
\hline
\end{tabular}

of the data logger. The results of the spectrum were recorded and saved in the PC (Fig. 4).

The range in which one plant can detect a person's position is very short; about $1.5 \mathrm{~m}$ is the maximum. In this paper, two plants are used, and the overlapping area that each plant can detect is also small. Thus, three locations are sufficient for the experiment. If we want to detect more locations, we need to use more plants.

The bioelectric potentials were measured at a sampling rate of $512 \mathrm{~Hz}$ and transmitted to a PC by a data logger. The output of data points is a time sequence form of voltage. Sets of 512 data points were regarded as 1 unit of analysis data. For every 32-sampling point, there is 1 unit of 512 data samples processed (Fig. 5).

Next, the raw data are calculated to obtain parameters: cepstrum coefficients and average, minimum, and maximum values. The cepstrum is the result of the inverse fast Fourier transform (FFT), which is the power spectrum of the target signal. This yields the detailed target signal for excellent analysis. The procedure to obtain the cepstrum coefficient is as follows: (5)

1. Determination of $S(t)$ as a potential signal,

2. Determination of power spectrum $S(\omega)$ using FFT of $S(t)$ and square calculation,

3. Calculation of the value of $\log |S(\omega)|$,

4. Determination of cepstrum coefficient values by calculating the inverse FFT of $\log |S(\omega)|$.

On the basis of the calculation, we determined 29 parameters, 26 of which are cepstrum coefficients, and the maximum value, minimum value, and average from each signal. In total, we obtained 319 parameters for 11 blocks (Fig. 6). We chose six parameters or variables from the cepstrum values, which include three variables from each of two plants for every object position.

\subsection{Generation of rules}

These rules are generated from the positions of three objects. We determine the rules for which the minimum support is 0.10 and the minimum confidence is 0.50 . The rules are presented in Table 2.

According to Table 2, we see that there are positions of three objects from two plants providing bioelectric potentials. Each position has two rules based on the limitation of the minimum support and confidence values. "Att" stands for an attribute; there are six attributes in total. Each attribute is a representation of a signal from the bioelectric potential data set.

Every rule contains three components: the antecedent, the consequent, or none of them. Furthermore, every component consists of a lower bound, an attribute, and an upper bound. An antecedent is the precondition that is followed by the consequent as its conclusion. In other 


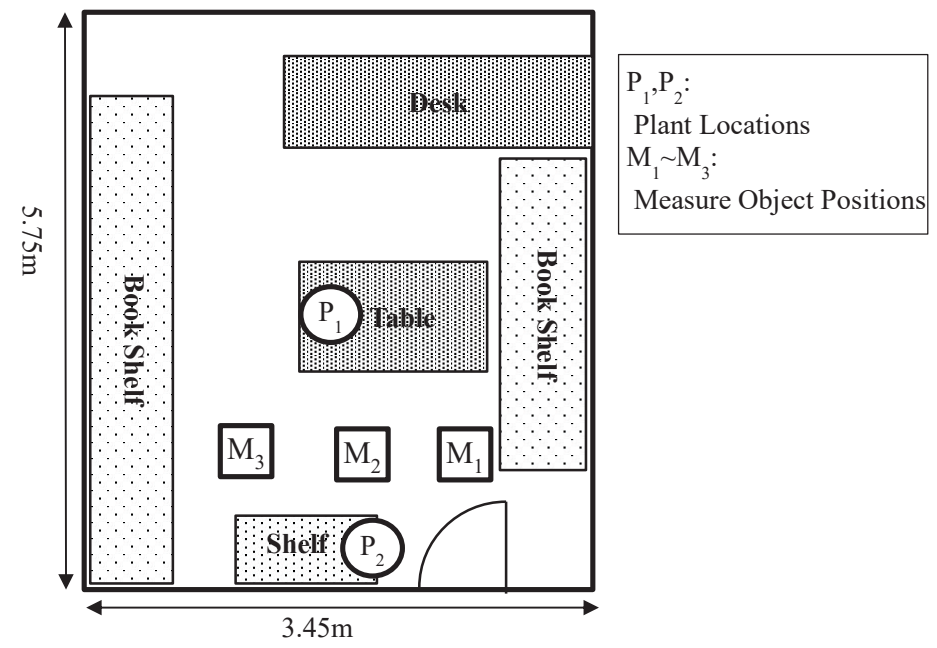

Fig. 4. Experimental environment. ${ }^{(5)}$

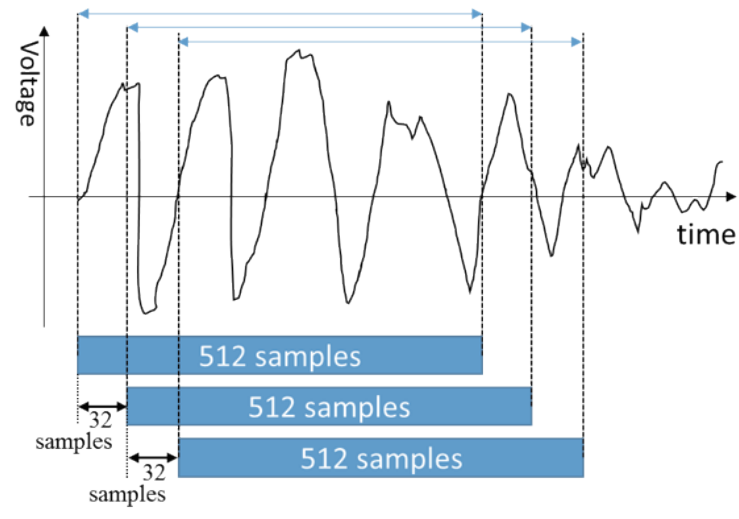

Fig. 5. (Color online) Sampling period for bioelectric potential data set. ${ }^{(5)}$

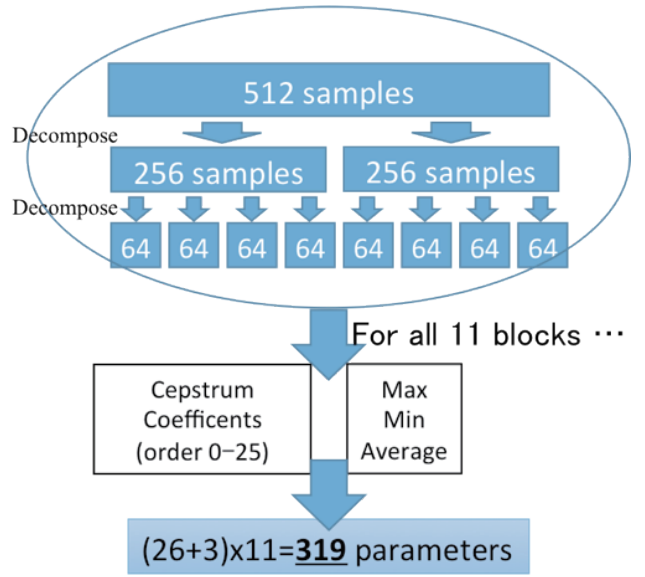

Fig. 6. (Color online) Parameter determination from a unit of analysis. ${ }^{(5)}$

Table 2

Representation of rules.

\begin{tabular}{|c|c|c|c|}
\hline & Position 1 & Position 2 & Position 3 \\
\hline \multirow{7}{*}{ Rule 1} & Antecedents & Antecedents & Antecedents \\
\hline & $1.733722<$ Att $2<1.760375$ & $1.751314<$ Att $2<1.874325$ & $4.566441<$ Att $1<4.583481$ \\
\hline & $1.234203<$ Att $6<1.268771$ & $5.044341<$ Att $4<5.094243$ & $1.053597<$ Att $3<1.223737$ \\
\hline & Consequents & Consequents & $4.846623<$ Att $4<4.861278$ \\
\hline & $4.632857<$ Att $4<4.692408$ & $1.791177<$ Att5 $<2.042655$ & Consequents \\
\hline & & $1.028409<$ Att $6<1.322651$ & $1.823191<\mathrm{Att} 2<1.935990$ \\
\hline & & & $1.815292<$ Att5 $<1.975540$ \\
\hline \multirow{6}{*}{ Rule 2} & Antecedents & Antecedents & Antecedents \\
\hline & $4.602205<$ Att $4<4.628170$ & $1.703965<$ Att $2<1.782008$ & $1.725530<$ Att $5<1.743504$ \\
\hline & $1.669055<$ Att $5<1.677074$ & $4.889498<$ Att $4<5.050735$ & $1.048957<$ Att6 $<1.111038$ \\
\hline & Consequents & $1.126966<$ Att $6<1.183972$ & Consequents \\
\hline & $1.124361<$ Att6 $<1.380390$ & Consequents & $1.738341<$ Att $2<1.846604$ \\
\hline & & $4.525496<$ Att $1<4.701028$ & \\
\hline
\end{tabular}


Table 3

Number of matching rules.

\begin{tabular}{lccc}
\hline & Position 1 & Position 2 & Position 3 \\
\hline Rule 1 & 1 & 1 & 0 \\
Rule 2 & 2 & 11 & 6 \\
\hline Total & 3 & 12 & 6 \\
\hline
\end{tabular}

words, we understand in a rule that "if antecedent then consequent". For example, for the first rule in position 1 , we can say that if att 2 and att6, then att 4 . In addition, all of the rules from each position are used for the matching process.

\subsection{Matching process and evaluation}

The aim of this process is to know the position of the human who walks around the object if there is a new data set (a testing data set). The testing data set, which is appropriate with one of the rules, indicates that a person is found around that object position.

First, we observed another data set at the first object position as well as explained in Sect. 3.2. Then, we use 6 variables of data set by choosing the first three of each two signal values. These variables are matched with the rules of each position extracted in Sect. 3.3. We counted how many times the values of the data set are matched to the rules of each position. Finally, the data set is classified to the position of the highest matching times. This is also done with other testing data sets from the second and third object positions. Finally, the result is a significant match. For all testing data sets in each position, the higher matching point was shown to be similar to the those of the original position (Table 3). Second, to ensure the matching step, we calculate the accuracy from each object position in 11 blocks. We tried to compare the rules extracted only from the 1st block with those from the other blocks. It is said that cepstrum coefficients represent the shape of frequency components of the signal. Therefore, we considered that the feature is similar if the behavior is observed in the signal even if the length of a signal is not the same. The evaluation resulted in almost $75 \%$ accuracy.

This research is more promising than previous studies that could not determine the object position accurately. They only detected the distance and estimated and classified the people as present globally. ${ }^{(5,6)}$

\section{Conclusions}

The purpose of this research was to determine the position of people using the bioelectric potential of plants. This has been successfully achieved. Using association analysis and a PSO approach, MOPAR has performed the estimation of the location of a person in one of three positions with an accuracy of approximately $75 \%$. In the future, we will improve the accuracy by other methods and other approaches such as by applying a modified MOPAR method, time series model, or deep learning analysis. 


\section{Acknowledgments}

This work was supported by JSPS KAKENHI Grant No. 17K00783 and Scholarship of KU Japan and DG-RSTHE Indonesia.

\section{References}

1 T. Shimbo and T. Oyabu: IEEJ Trans. Sens. Micromachines 124 (2004) 470.

2 K. Nomura, H. Nambo, and H. Kimura: IEEJ Trans. Sens. Micromachines 134 (2014) 206. doi:10.1541/ieejsmas.134.206

3 H. Nambo and H. Kimura: 10th Int. Conf. Management Science and Engineering Management (2017).

4 H. Nambo: APIEMS (2015) 1896.

5 H. Nambo and H. Kimura: Sens. Mater. 28 (2016) 369.

6 X. Jin: APIEMS (2014) 602.

7 K. Nomura, H. Nambo, and H. Kimura: IEEJ Trans. Sensors Micromachines 134 (2014) 206. doi:10.1541/ieejsmas.134.206

8 J. Tang, G. Zhang, B. Lin, and B. Zhang: A Hybrid PSO / GA Algorithm for Job Shop Scheduling, Int. J. (2010) 566.

9 J. S. Lee, S. Lee, S. Chang, and B. H. Ahn: Artif. Intell. Knowl. Eng. Appl. A Bioinspired Approach, Pt 2, Proc. 3562 (2005) 221.

10 W.-P. Lee and Y.-T. Hsiao: Inf. Sci. (Ny). 188 (2012) 80. doi:10.1016/j.ins.2011.11.020

11 V. Beiranvand, M. Mobasher-Kashani, and A. Abu Bakar: Expert Syst. Appl. 41 (2014) 4259. doi:10.1016/jeswa2013.12.043

12 J. Kennedy and R. Eberhart: Proc. IEEE Int. Conf. 4 (1995) 1942-1948.

13 Y. Xinjie and M. Gen: Introduction to Evolutionary Algorithms (Springer, London, 2010). doi:10.1017/CBO9781107415324.004

\section{About the Authors}

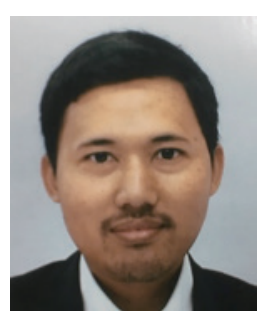

Imam Tahyudin was born in Indramayu, West Java, Indonesia. He is a Ph.D. student in the graduate school of NST in the division of Electrical Engineering and Computer Science in Kanazawa University, Japan. He has been a lecturer in STMIK AMIKOM Purwokerto, Central Java, Indonesia since 2009. He is a member of the Institute of Advanced Engineering and Science (IAES); the Association of Higher Education of Computer Science, Indonesia (APTIKOM); the Indonesian Computer, Electronics, Instrumentation Support Society (IndoCEISS); the Association of Information Systems (AIS); and the Association of Information Systems for Indonesia (AISINDO).

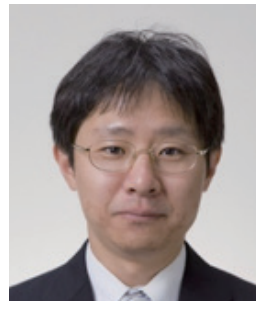

Hidetaka Nambo received his Ph.D. degree in 1999 from Kanazawa University. He was a Research Associate in 1999 in the Department of Electrical Information, Engineering Faculty of Kanazawa University. He has been a lecturer in the Graduate School of Natural Science and Technology since 2015. He is an associate professor in the College of Science and Engineering. He is engaged in research on monitoring systems by living plants and data mining. He is a member of the IEEE, the IEE of Japan, the IEICE of Japan, and the IPSJ of Japan. He can be reached at nambo@blitz.ec.t.kanazawa-u. ac.jp. 